\title{
Recruiting participants for injury studies in emergency departments
}

\section{Denise Kendrick, Ronan Lyons, Nicola Christie, Elizabeth Towner, Jonathan Benger, Lindsay Groom, Frank Coffey, Phillip Miller, Rachel Murphy for The UK Burden of Injury Study Group}

Emergency departments have the potential to maximize recruitment efficiency and minimize recruiting costs

See linked article, p 88

$\mathrm{F}$ or many studies, especially those requiring incident injury cases, emergency departments are the most suitable location for recruiting participants. ${ }^{1}$ Although the total number of injury attendances is greater in outpatient or primary care settings than in emergency departments, ${ }^{2}$ geographical spread and the mixture of incident and prevalent cases make recruiting participants from these sites less feasible, more time consuming and more costly. Emergency departments, on the other hand, will see the largest number and spectrum of injury cases, usually presenting very shortly after injury in a single healthcare setting. This has the potential to maximize recruitment efficiency and minimize recruiting costs. Recruiting in emergency departments also provides opportunities to study the aetiology and epidemiology of injuries before recall of events diminishes with time and to enroll participants for studying short- and longterm consequences of injury. They are the only setting in which complete ascertainment of incident cases of specific injuries may be possible-for example, virtually all patients with long bone fractures will attend an emergency department, fewer will attend primary care and a proportion will be admitted to hospital, but this will vary between hospitals depending on a range of factors including clinician preference for management options, bed availability, social circumstances. ${ }^{34}$ Recruiting cases from emergency departments therefore, has the potential to minimize the selection bias inherent in recruiting such cases from other sites.

\section{WHY CAN IT BE DIFFICULT TO RECRUIT STUDY PARTICIPANTS IN EMERGENCY DEPARTMENTS?}

Inspite of the potential for recruiting injured patients to studies within emergency departments, there are characteristics of the clinical setting and of injured patients which may make recruitment difficult. Emergency departments are often busy and crowded places, and the demand for emergency care continues to increase. ${ }^{5}$ Many emergency departments experience shortfalls in medical staffing and difficulties in recruiting and maintaining skilled nursing staff, certainly within the UK. ${ }^{6}$ Shift work and national and international workforce initiatives, which impact on work patterns of junior medical staff (eg, the UK Modernising Medical Careers Foundation Programme, which allows junior medical staff to experience a wide range of specialties for 4-month periods, ${ }^{7}$ and the European Working Time Directive), mean that a large number of staff, a significant proportion of whom will change over a relatively short period of time, may need to be involved in recruiting patients.

Many injured patients do not spend long periods of time in emergency departments, indeed in England the government set a target that by 2004 no one should wait more than $4 \mathrm{~h}$ in an emergency department from arrival to admission to a bed, transfer or discharge. Recent data suggest that only 5\% of patients exceed this waiting time, with smaller figures for more minor injuries. ${ }^{6}$ Hence, there may be only a small time window within which participants can be recruited. Emergency department patients can also have complicated journeys, moving frequently both within and outside of the department (eg, from waiting room to trolley while waiting for cubicle, to cubicle, to $x$ ray, to plaster room and then discharged), so researchers may have difficulty finding where the patient is and may have little time to discuss the study before the patient is moved to another clinical area. Furthermore, most emergency department attendances are single encounters, ${ }^{6}$ so there is no ongoing patient-clinician relationship and patients may feel little affiliation with, or allegiance to the department, which may affect their willingness to participate in research. Finally, emergency department attenders who perceive themselves to be "at fault" for their injury, or for it to be in some way "self-inflicted" may be less willing to participate in research studies.

There are some specific injuries that may be more difficult to recruit to studies than others. This includes rare injuries, such as those in the farm workers in the study by Day et al, ${ }^{8}$ those where once serious injury has been excluded the patient gets discharged very quickly-for example, minor head injuries-and those where the patient's condition or management makes the physical process of recruitment difficult-for example, those who are immobilized who may find reading information sheets difficult (eg, suspected neck injuries) or would have difficulty signing consent forms (eg, dominant hand or arm injuries). Patients who have had serious injury are often not well placed to give valid, informed consent immediately afterwards: they may be frightened, in pain or under the influence of powerful medications. ${ }^{9}$ In some cases, they are too incapacitated to understand or retain information and may even be deeply unconscious.

\section{HOW DO PRIVACY LEGISLATION, ETHICAL AND RESEARCH GOVERNANCE REQUIREMENTS AFFECT RECRUITING IN EMERGENCY DEPARTMENTS?}

A combination of privacy legislation, ethical or research governance requirements may affect recruiting participants from emergency departments in several ways. Firstly, the requirement for healthcare providers to make the first approach to potential participants prevents researchers from identifying potential participants in the department or making the first approach themselves. It is argued that this benefits the patient as health information, such as knowledge of a particular injury or of attendance at a healthcare facility is not divulged by healthcare providers to researchers. ${ }^{10}$ The other potential patient benefit is that it may be easier for patients to decline participation in a study when the first approach is made by someone other than a member of the research team. As this system relies on healthcare staff, whose prime concern is patient care, to identify potentially eligible participants and to make the first approach, it is unsurprising that achieving recruitment targets is difficult in these circumstances.

The second and closely related issue is that of the (not necessarily universal) ${ }^{11}$ requirement for "opt-in" systems for recruitment, of particular relevance for 
non-face-to-face methods, such as postal recruitment. Under the "opt-in" approach, researchers are only allowed to contact potential participants who respond positively to a request that they can be contacted by the researcher, from a member of the healthcare team. There is evidence that "opt-in" approaches are associated with lower response and/or recruitment rates, ${ }^{812} 13$ and hence, with increased research costs. If lower recruitment rates result in failing to achieve the required sample size, this is also an ethical issue, as Crombie points out, "Research should only be undertaken when there is a high likelihood of producing valid findings. Ethical requirements which result in invalid research may themselves be unethical". ${ }^{14}$ "Opt-in" approaches have been found to be associated with response or recruitment biases related to age, gender, residence in deprived areas, ethnic group, preferred language, mortality rates, length of hospital stay, level of consciousness, family history of disease and in preferred healthcare decision-making roles. ${ }^{12}{ }^{13}$ Such biases may result in under-recruitment of those at greatest risk or those who may benefit most from an intervention, inaccurate estimation of the incidence or prevalence of conditions, a reduction in power to detect subgroup differences and limited generalizability of the findings. ${ }^{10}$ "Opt-in" systems may, therefore, be achieving patient confidentiality at the expense of scientific rigor.

How concerned are patients about the confidentiality of their health information in relation to research? There is little evidence on this to date, but a recent large survey of the British public found $87 \%$ would not consider it an invasion of their privacy by the National Cancer Registry if they sent them a letter asking them to take part in a research study. ${ }^{16}$ Other studies have found only a very small number of potential participants queried or complained about a researchers' right to approach them and most respondents in an Australian survey were in favor of health databases being used for research purposes. ${ }^{81718}$

Clearly, a balance has to be struck between the patient's right to confidentiality and the benefits of medical research to society. The UK's Academy of Medical Sciences has joined this debate with their recent report on using health information in medical research. This outlines the benefits of medical research to society and acknowledges that it is increasingly being inhibited by "inappropriate constraints on the use of personal health data". ${ }^{19}$ It makes a series of recommendations on interpreting the legal framework, improving regulatory pro- cesses, developing good practice in research using personal data, harnessing the opportunities provided by new National Health Service information technology and engaging the public. Although this is a UK document, the recommendations will be relevant to other countries with similar legislative, ethical and research governance arrangements. Other recent suggestions to facilitate research within the National Health Service include making "its research mission explicit when people use the service, in the same way that patients attending a teaching hospital are informed about and invited to participate in the professional education that happens there" ${ }^{20}$

Finally, the issues surrounding the recruitment of incapacitated patients to research studies are particularly pertinent to emergency departments. These have been addressed in relation to clinical trials in varying ways in different countries. In the US, a waiver of consent allows researchers to enroll incapacitated patients into trials provided that they fulfill trial entry criteria, although this position is currently under review. ${ }^{21}$ In the European Union (EU), the EU Directive 2001/20/EC requires prior informed written consent before subjects can be recruited to clinical trials of medicinal products. This has led to substantial difficulties in establishing highquality trials in emergency care ${ }^{22}$ and the development of recommendations for changes to the EU Directive to allow such research to proceed under clearly defined conditions. $^{23}$ In the UK, a suitable legal framework now exists, under the Medicines for Human Use (Clinical Trials) Regulations 2004. This allows a personal legal representative or professional legal representative to consent on behalf of an incapacitated patient, ${ }^{24}$ but the framework seems to be inconsistently applied, with areas of potential confusion and a lack of central guidance. ${ }^{25} 26$ Although this framework should prove helpful for clinical trials, recruiting incapacitated patients to other study designs may still prove problematic.

\section{HOW CAN RECRUITMENT TO RESEARCH STUDIES IN EMERGENCY DEPARTMENTS BE MAXIMIZED?}

Here, we draw on our experience in recruiting more than 1000 injured patients to the UK Burden of Injury Study, which is a prospective cohort study measuring injury-related disability in patients aged $\geqslant 5$ years presenting with a wide range of injuries to four emergency departments in the UK. Our experience suggests that the following strategies may be helpful:
- It is very important to build and maintain good relationships with emergency department staff. Involving emergency department staff in the design of the study in the early stages will increase their ownership of it. Raising awareness of the study and offering repeated briefing sessions for the staff are necessary. In large departments, it may take several months to get to meet all relevant staff and this time needs to be built into the project. Debriefing sessions with emergency department staff are helpful to refine recruitment methods, tailor them to local requirements, identify any problems and agree on locally appropriate solutions.

- Use dedicated researchers based in the emergency department to undertake recruiting. ${ }^{27}$ Researchers with experience in undertaking clinical work in the department that they will be recruiting in will know how the department works, what clinical and information technology systems are in place and will already be known to the emergency department staff.

- Maintain a presence in the department. It is important that staff get to know the researchers and that the researchers are there when potentially eligible participants are in the emergency department, including evenings and weekends. Although this may seem to entail a lot of waiting around for the researcher, relying on busy emergency department staff to notify (absent) researchers when potentially eligible patients are in the department is unlikely to be effective. Computerized systems for notifying researchers when eligible patients are in the department have been found to be effective in increasing recruitment in previous studies, ${ }^{28}$ but this has not been feasible in the UK Burden of Injury Study.

- Even if dedicated research staff are used to recruit participants, healthcare staff will still spend time on the research study. Acknowledge and pay for the time spent by emergency department staff on research studies and build such costs into research proposals.

- Timing of recruitment. There are times when the emergency department is too busy and the staff do not have time to approach potential participants. Recruiting at slightly less busy times may be more effective.

Day et $\mathrm{al}^{\prime} \mathrm{s}^{8}$ modified recruitment method, of using either a written "optin" or "opt-out" letter sent by the hospital was also considered by our study team. We sought ethical approval to use a written 
"opt-in" letter sent by the emergency department consultant, but approval was not granted on the grounds that it might have alerted family members to the patient's attendance at the emergency department. Such a decision is concerning as inability to use even an "opt-in" letter will only serve to make recruitment in emergency departments more difficult.

\section{CONCLUSION}

Recruiting injured patients to research studies in emergency departments has particular challenges due to the nature of the clinical setting and of the patients that present to emergency departments. Current privacy legislation, and ethical and research governance requirements have the potential to reduce participation rates and to bias study populations. Practical experience of recruiting participants in emergency departments suggests that recruitment can be successful, but that, where healthcare providers have to make the first approach to patients, the (not inconsiderable) costs of using this approach need to be incorporated into research proposals. The difficulties faced by researchers in using personal health data are being acknowledged and recent guidance from the Academy of Medical Sciences should help facilitate such research.

Injury Prevention 2007;13:75-77.

doi: 10.1136/ip.2006.013730

\section{Authors' affiliations}

Denise Kendrick, Lindsay Groom, Rache

Murphy, Division of Primary Care, University Park, Nottingham, UK

Ronan Lyons, Centre for Health Information, Research and Evaluation (CHIRAL), School of Medicine Swansea University, Swansea, UK Nicola Christie, Surrey Injury Research Group, Postgraduate Medical School, Daphne Jackson Road, Manor Park, University of Surrey, Guildford, UK
Elizabeth Towner, Centre for Child and Adolescent Health, Faculty of Health and Social Care, University of the West of England, Hampton House, Cotham Hill, Bristol, UK Jonathan Benger, Centre for Clinical and Health Services Research, Faculty of Health and Social Care, University of the West of England, Glenside Campus, Bristol, UK

Frank Coffey, Phillip Miller, Emergency Department, Nottingham University Hospitals NHS Trust, Queen's Medical Centre Campus, Nottingham, UK

Correspondence to: Dr D Kendrick, Division of Primary Care, Floor 13, Tower Building, University Park, Nottingham NG7 2RD, UK; denise.kendrick@nottingham.ac.uk

Accepted 5 December 2006

Competing interests: None.

\section{REFERENCES}

1 Lyons RA, Brophy S, Pockett R, et al. Purpose, development and use of injury indicators. Int J Ini Prev Saf Promot 2005:4:207-11.

2 Bishai D, Gielen A. How much outpatient care is provided for injuries? Inj Prev 2001;7:70-3.

3 Walsh SS, Jarvis SN. Measuring the frequency of "severe" accidental injury in childhood. J Epidemiol Community Health 1992;46:26-32.

4 Lyons RA, Polinder S, Larsen CF, et al Methodological issues in comparing injury incidence across countries. Int J Inj Prev Saf Promot 2006; 13:63-70.

5 Department of Health. Reforming emergency care London: $\mathrm{DH}, 2001$

6 National Audit Office. Improving emergency care in England, Report by the Comptroller and Audito General.HC 1075 Session 2003-2004. London: Stationery Office, 2004.

7 NHS. Modernising Medical Careers: foundation programmes http://www.mmc.nhs.uk/pages/ foundation (accessed 11 Sep 2006).

8 Day L, Langley J, Stathakis V, et al. Challenges of recruiting farm injury study participants through hospital emergency departments. Inj Prev 2007.

9 Gammelgaard A, Mortensen OS, Rossel P. Patients' perceptions of informed consent in acute myocardial infarction research: a questionnaire based survey of the consent process in the DANAMI-2 trial. Heart 2004;90: $1124-8$.

10 Hewison J, Haines A. Overcoming barriers to recruitment in health research. BMJ 2006;333:300-2.

11 Pegg MS. Overcoming barriers to recruitment in health research: Some research ethics committees believe in facilitating ethical research. $B M J$ 2006;333:300-2

12 Trevena L, Irwig L, Barratt A. Impact of privacy legislation on the number and characteristics of people who are recruited for research: a randomised controlled trial. J Med Ethics 2006;32:473-7.

13 Tu JV, Willison DJ, Silver FL, et al. Impracticability of informed consent in the Registry of the Canadian Stroke Network. N Engl J Med 2004:350:1414-21

14 Crombie IK, McMurdo MET, Irvine L, et al Overcoming barriers to recruitment in health research: concerns of potential participants need to be dealt with. BMJ 2006:333:398.

15 Angus V, Entwistle V, Emslie M, et al. The requirement for prior consent to participate on survey response rates: a population-based survey in Grampian. BMC Health Services Res 2003;3:21.

16 Barrett G, Cassell JA, Peacock JL, et al. National survey of British public's views on use of identifiable medical data by the National Cancer Registry. BM 2006;332:1068-72

17 Inskip HM, Godfrey KM, Robinson SM, et al. Cohort profile: the Southampton Women's Survey. Int J Epidemiol 2006;35:42-8.

18 Whiteman DC, Clutton C, Hill D. Australian public's views on privacy and health research. $B M J$ 2006:332:1274

19 Academy of Medical Sciences. Personal data for public good: using health information in medical research. London: Academy of Medical Sciences, 2006, http://acmedsci.ac.uk/images/project/ Personal.pdf (accessed 13 Feb 2007).

20 Souhami R. Governance of research that uses identifiable personal data. BMJ 2006;333:315-16.

21 Associated Press. Tell public about trauma tests, experts urge. http://ctv.ca/servlet/ArticleNews/ story/CTVNews/20061012/ trauma experts $061012 / 20061012 \mathrm{hub}=$ Health (accessed 13 Feb 2007)

22 Coats TJ, Ng G, Shakur H. Consent in emergency research. Emerg Med J 2006;23:489-490.

23 Liddell K, Chamberlain D, Menon D, et al. The European Clinical Trials Directive revisited: the VISEAR recommendations. Resuscitation 2006;69:9-14

24 Office of Public Sector Information. Medicines for Human Use (Clinical Trials) Regulations 2004 http://legislation.hmso.gov.uk (accessed 26th October 2006)

25 Coats TJ, Shakur H. Consent in emergency research: new regulations. Emerg Med J 2005;22:683-5.

26 College of Emergency Medicine. Position statement on Consent in Emergency Care Research, 2006. http://emergencymed.org.uk/temp/1541position-ethics.doc

27 Isaacman D, Reynolds EA. Effect of a research nurse on patient enrollment in a clinical study. Pediatr Emerg Care 1996;12:340-2.

28 Weiner DL, Butte AJ, Hibberd PL, et al. Computerized recruiting for clinical trials in real time. Ann Emerg Med 2003;41:242-6. 